\title{
What is happening at Lab Animal
}

\author{
Introducing a new online platform for the journal, new types of content, and a new Chief Editor.
}

\begin{abstract}
A t Lab Animal, we strive to be a leading scientific publication dedicated to in vivo science and technology that advances basic understanding and use of model organisms. We publish basic research methods and technologies and also cover important news, business, and regulatory matters that impact the development and application of model organisms for preclinical research.

While being proud of our long publishing history in this space, in recent years we have made significant changes to our content and publishing platforms to ensure we deliver a product that is of high quality to our readers and authors. In 2017, we migrated the journal from its old website and onto the Nature Research platform on nature. com. This move enabled us to improve the reader's experience by providing our content on a more reliable-and modern-platform, and it facilitated aligning our journal's editorial practices and policies with the rest of the Nature Research journal portfolio.

While we have always sought to generate content that we believe best serves our readership, science evolves and so do we. We have recently made some changes to our content types that we hope will be
\end{abstract}

well received by our community. We have added the Comment article type, which is intended to be a forum for our readers to interact with us and express views and opinions on topics of interest to our audience. Starting this month, we also introduce a new article type to report on methods: the Protocol.

The Protocol article type will follow the format published at our sister journal, Nature Protocols, and will be a peer reviewed, step-by-step set of instructions on previously published techniques. We will focus on Protocols relevant to animal model research, covering a variety of organisms and research practices. The emphasis of these papers will not be the novelty of the methodology but the practical value of the 'recipe' and its capacity to be used as a resource by scientists interested in implementing the method in their labs. Protocols will also provide background information about how the method was developed and how it compares to other techniques, as well as discuss the method's limitations. Protocols will provide important and detailed information on reagents, warnings and troubleshooting. With the Protocol, we wish to 'ease the pain' associated with setting up a new technique or experimental approach in your lab. Additionally, we think that the Protocol article type will help fulfill a need in today's science for increased reproducibility, reliability, and robustness in animal research. An additional benefit includes a better return on research dollars.

Finally, Dustin Graham, our former Chief Editor, took a position at BMC Medicine, and we want to take this opportunity to introduce our new Chief, Clark Nelson. Clark earned his PhD in mass spectrometry and protein identification and quantitation at the University of Wisconsin Cellular and Molecular Biology program, later moving to the School of Veterinary Medicine at the University of Wisconsin to study small molecule changes in mammalian hibernation. Throughout his research career, Clark has developed a familiarization and love for animal research, including protocols and institutional care and use policies, and is excited to serve and champion the journal in this new stage.

Published online: 24 September 2018 https://doi.org/10.1038/s41684-018-0158-9 